\title{
Is improved fitness associated with reduced pain intensity in patients with low back pain?
}

\author{
Cecilie Rud Budtz ${ }^{1 *}$, Lene Wulff Krogsgaard ${ }^{1}$, Berit Schiøttz-Christensen ${ }^{2}$ and Thomas Maribo ${ }^{3,4}$ \\ ${ }^{1}$ Central Region, Denmark \\ ${ }^{2}$ Spine Centre of Southern Denmark, Hospital Lillebaelt, Middelfart and Institute of Regional Health Research, University of Southern Denmark, Odense, Denmark \\ ${ }^{3}$ Department of Public Health, Section of Clinical Social Medicine and Rehabilitation, Aarhus University, Denmark \\ ${ }^{4}$ DEFACTUM, Central Denmark Region Aarhuc, Denmark
}

\begin{abstract}
Introduction: Low back pain (LBP) is a common global health problem and it causes more disability worldwide than any other disease. Despite this, knowledge concerning causes, prevention and effective treatments is lacking. It is a general assumption that LBP is associated with reduced physical capacity, but only few studies have examined this association.

Objective: To examine the association between fitness and pain intensity in LBP-patients. Further to examine the association between clinically relevant improved fitness and clinically relevant reduced pain intensity at follow-up based on the patients' baseline fitness levels. The hypothesis being that the association is most substantial for patients with poor baseline fitness levels at inclusion.

Methods: Cohort study with three months' follow-up based on data from a previous RCT study. The study population consisted of 176 patients with LBP and complete 3 months' follow-up on fitness and pain. Clinically relevant improved fitness was defined as improved fitness $\geq 10 \%$ from baseline to follow-up. Clinically relevant change in pain intensity was defined as a reduction $\geq 30 \%$ on the Numerical Rating Scale. The association was examined by Spearman correlation and logistic regression and presented by OR $(95 \mathrm{CI})$.
\end{abstract}

Results: No correlation between fitness and pain intensity at baseline was found. Also, no association between clinically relevant improved fitness and clinically relevant reduced pain intensity based on the patients' baseline fitness levels was detected.

Conclusions: The study examined the association between fitness and pain intensity in LBP-patients and no association between changes in fitness and pain intensity during the 3 months' follow-up was seen. The study contributes with evidence based on clinically relevant changes in fitness and pain intensity.

\section{Introduction}

Low back pain (LBP) is a common global health problem causing more disability worldwide than any other disease and is one of the leading causes to absenteeism [1]. In-spite of high prevalence and the costs associated hereby there is a lack of knowledge concerning causes, prevention and effective treatments [2,3]. There is no explicit consensus regarding treatment of LBP and the concepts of interventions vary from very simple to extensive multidisciplinary biopsychosocial rehabilitation programs.

A review from 2014 provided moderate to low quality evidence that multidisciplinary biopsychosocial rehabilitation is more effective than usual care or physical treatment in terms of pain, disability and work status [4]. The comparison between multidisciplinary biopsychosocial rehabilitation and physical treatment is complicated as there is a lack of evidence of the effects of physical treatment and the quality of existing evidence is low [4]. To enhance the quality, it is recommended to investigate the effectiveness of different types of physical treatment $[5,6]$.

It is a general assumption that LBP is associated with reduced physical capacity and fitness or maximum oxygen uptake $\left(\mathrm{VO}_{2} \max \right)$ is often used as an approximation for physical capacity. This assumption has been examined with varying results [7-13].

Few studies have examined the association between fitness and
LBP with divergent results $[7,9,11,12]$. One study found an association between improved fitness and reduced pain [9] but other studies have not been able to detect this association [7,11-14].

As the association between fitness and pain intensity remains unclear further studies are required. The intention of the current study is to provide evidence on the association between fitness, as one aspect of physical capacity, and the intensity of pain.

The aim of the study was to examine the association between fitness and pain intensity in patients with LBP. Further to examine the association between clinically relevant improved fitness and clinically relevant reduced pain intensity during follow-up based on the patients' baseline fitness levels. The hypothesis being that the association would be most substantial for patients with poor baseline fitness levels at inclusion.

Correspondence to: Cecilie Rud Budtz, Central Region, Denmark, E-Mail: cecilie.budtz@hotmail.com

Key words: low back pain, fitness, VO2 max, clinical relevance

Received: October 19, 2016; Accepted: November 03, 2016; Published: November 07, 2016 


\section{Methods}

\section{Design}

The study was a cohort study with three months' follow-up based on available data from a previously RCT study [15].

The aim of the original RCT study was to assess if counseling by an occupational physician addressing experienced workplace barriers and physical activity integrated as a part of LBP outpatient treatment influenced pain, function and sick leave. At baseline and follow-up, the patients completed different physical tests and filled out questionnaires including pain scores. Neither the control nor the intervention group received interventions aimed at improving their fitness, but all the patients received brief instructions in exercises. The intervention group also received counseling sessions including goals for physical activities generally consisting of $3 \times 45$ min a week of moderate intensity.

The inclusion criteria in the original RCT study were; patients aged 17-63 with paid work employment and expressed concerns about their ability to maintain job independently of sick leave status. Exclusion criteria were; patients referred to surgery, pregnancy or severe comorbidity.

In the original RCT study 300 patients were allocated to the intervention $(n=150)$ or control group $(n=150)$. The study population for the present study included 176 patients with complete follow-up on fitness and pain intensity (Figure 1).

\section{Fitness}

Fitness was calculated as $\mathrm{ml} /$ oxygen $/ \mathrm{min} / \mathrm{kg}$ estimated using Aastrands cycle test [16]. Aastrands cycle test is a submaximal test and the maximum oxygen uptake was estimated from approximately 10 minutes of cycling. As weight is incorporated in fitness, $\mathrm{VO}_{2}$ max is consequently presented in the analysis in addition to fitness to demonstrate if changes in fitness are a result of changes in weight.

To incorporate the patients baseline fitness levels, the patients were grouped into four fitness categories very low, low, medium and high/

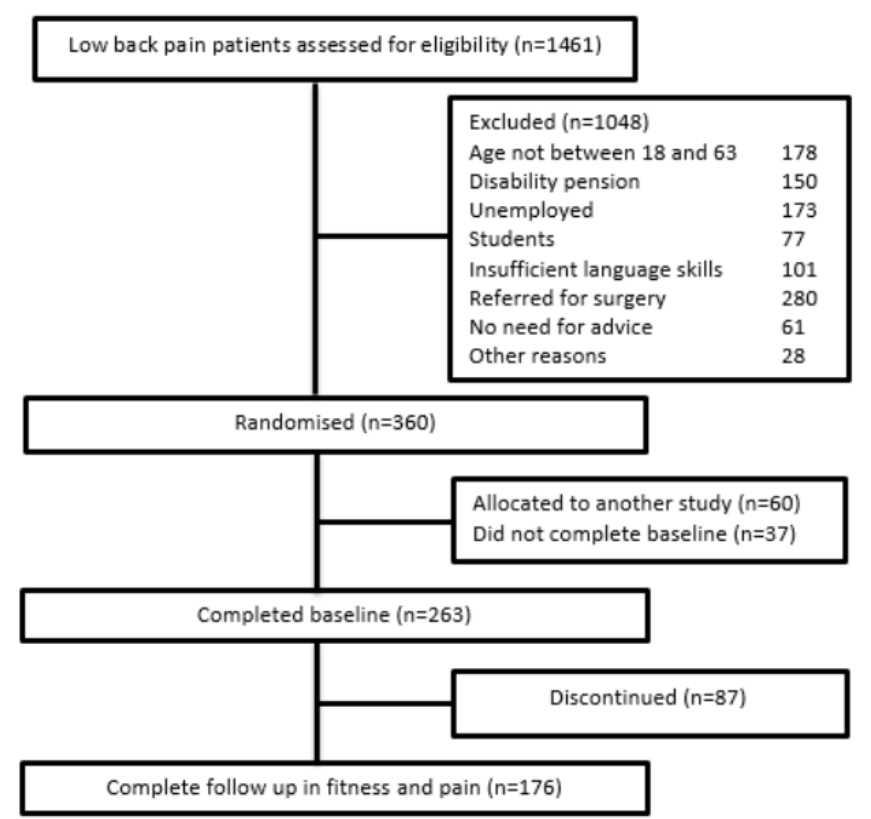

Figure 1. Flowchart of the selection of the study population. very high based on their baseline fitness, gender and age [17].

Clinically relevant improved fitness was defined as an improvement from baseline to follow-up on $10 \%$ or more. No literature was found to determine the threshold, and $10 \%$ was based on clinical reasoning. A 10\% improvement in fitness was considered perceptible for the individual patient. Within each fitness category the group with improved fitness of $\geq 10 \%$ was compared to the group with an improvement $<10 \%$.

\section{Pain}

Pain intensity was assessed using the Numerical Rating Scale (NRS). NRS is a numerical scale with 11 points reflecting the patients experienced pain intensity. The pain intensity is scored from 0 ; no pain to 10; worst pain imaginable, and the pain score represent the patients mean pain intensity during the last 3 months. Clinically relevant reduced pain intensity was defined as a reduction from baseline to follow-up on $30 \%$ or more based on practical guidance regarding the minimal important change on frequently used measures of pain and functional status for LBP developed by Ostelo et al. [14].

\section{Statistical analysis}

The statistical analysis was conducted in STATA 14.1 (StataCorp LP, Texas, USA).

Baseline characteristics of the study population and the group who was lost to follow-up was compared using the Mann-Whitney rank-sum test. Spearman correlation evaluated the strength of the association between baseline fitness and pain.

A boxplot visualized pain intensity at baseline and follow-up in the four fitness categories. The Kruskal-Wallis test was used to analyze differences between the four fitness groups at baseline as well as changes after 3 months in fitness, $\mathrm{VO}_{2} \max$ and pain intensity. The association between clinically relevant improved fitness and reduced pain intensity was analyzed in the four fitness categories by logistic regression and presented as odds ratio (OR) and 95\% confidence interval (95 CI).

\section{Results}

Table 1 presents the baseline characteristics of the included study population $(n=176)$ and the group who was lost to follow-up ( $n=87$, $49 \%$ ). There were no significant differences between the groups in the measured variables.

Spearman correlation between absolute values of baseline pain intensity and fitness was $-0,13(p=0.09)$, meaning no correlation between baseline values of fitness and pain was detected.

The patients were grouped into four fitness categories to examine

Table 1. Baseline characteristics of the study population compared to the group who was lost to follow up.

\begin{tabular}{|l|c|c|c|}
\hline & Study population & Lost to follow-up & $\mathbf{p}^{*}$ \\
\hline N & 176 & 87 & \\
\hline Gender female/male (\%) & $55 / 45$ & $55 / 45$ & $0.98^{\dagger}$ \\
\hline Age & $46[37 ; 53]$ & $45[37 ; 53]$ & 0.85 \\
\hline Fitness & $25,5[23.3 ; 29.0]$ & $26.0[23.0 ; 30.0]$ & 0.49 \\
\hline VO max & $30.0[23.9 ; 34.9]$ & $28.4[23.4 ; 35.5]$ & 0.87 \\
\hline NRS (0-10) & $2.2[1.8 ; 2.9]$ & $2.3[1.9 ; 2.7]$ & 0.5 \\
\hline
\end{tabular}

Values are median [IQR] unless stated otherwise

"Tested using the Mann-Whitney rank-sum test

†Tested using Pearson’s chi-squared 
the association for the patients with poorest baseline fitness levels. Figure 2 shows pain scores at baseline and follow-up in the four fitness groups.

Table 2 presents baseline characteristics and the patients' changes in fitness, $\mathrm{VO}_{2}$ max and pain scores in the four fitness categories. The group with very low fitness at baseline had the highest baseline pain score and naturally the lowest fitness score. Compared to the other fitness groups the very low group had the highest proportion of patients achieving an improvement in fitness and reduction in pain intensity during the three months.

Incorporating thresholds for clinically relevant changes in fitness (10\%) and pain intensity (30\%) showed no significant association between improved fitness and reduced pain in either of the four fitness categories (Table 3).

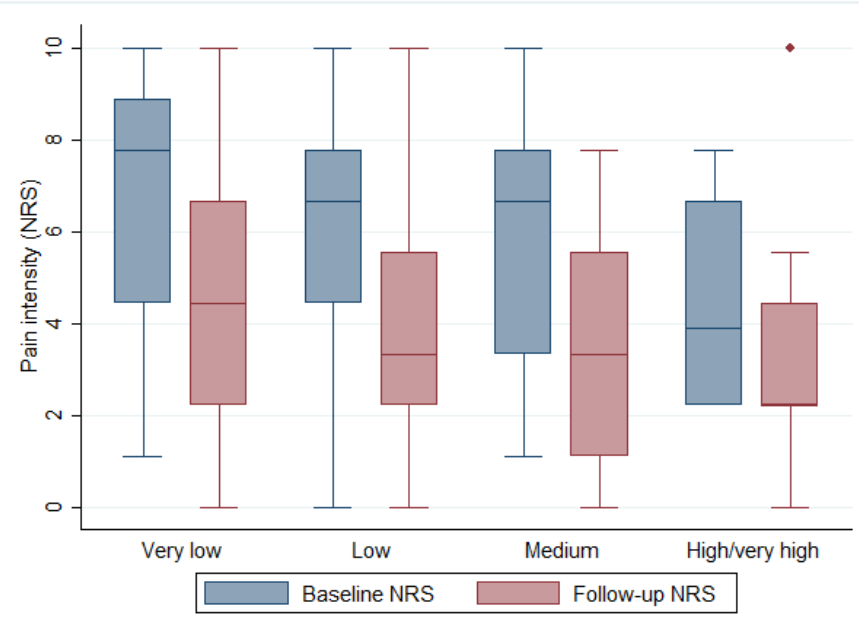

Figure 2. Pain scores (NRS 0-10) presented as median, IQR and range at baseline and follow-up in the four baseline fitness categories.

Table 2. Included patients baseline characteristics and changes in fitness, $\mathrm{VO}_{2}$ max and pain intensity presented in the four fitness categories.

\begin{tabular}{|c|c|c|c|c|c|}
\hline & Very low & Low & Medium & $\begin{array}{l}\text { High/very } \\
\text { high }\end{array}$ & $\mathbf{p}^{\dagger}$ \\
\hline $\mathbf{n}$ & 63 & 60 & 39 & 14 & \\
\hline Male (\%) & 62 & 32 & 38 & 43 & $<0.01^{*}$ \\
\hline Age & $48[41 ; 56]$ & $46.5[37 ; 51]$ & $45[40 ; 53]$ & $37[29 ; 50]$ & 0.06 \\
\hline \multicolumn{6}{|l|}{ Baseline } \\
\hline Fitness & $\begin{array}{l}21 \\
{[18.7 ; 25.4]}\end{array}$ & $\begin{array}{l}29.9 \\
{[27.7 ; 32.4]}\end{array}$ & $\begin{array}{l}35.7 \\
{[32.4 ; 38.6]}\end{array}$ & $\begin{array}{l}44.4 \\
{[42.6 ; 48.4]}\end{array}$ & $<0.01$ \\
\hline $\mathrm{VO}_{2} \max$ & $\begin{array}{l}1.9 \\
{[1.4 ; 2.2]}\end{array}$ & $2.2[1.9 ; 2.7]$ & $2.5[2.1 ; 3.2]$ & $3.1[2.8 ; 3.7]$ & $<0.01$ \\
\hline Pain & $\begin{array}{l}7.8 \\
{[4.4 ; 8.9]}\end{array}$ & $6.7[4.4 ; 7.8]$ & $6.7[3.3 ; 7.8]$ & $3.9[2.2 ; 6.7]$ & 0.02 \\
\hline \multicolumn{6}{|l|}{ Changes } \\
\hline Fitness & $\begin{array}{l}2.4 \\
{[0.3 ; 5.4]}\end{array}$ & $1.4[-1.2 ; 3.7]$ & $2.9[-0,5 ; 6.1]$ & $\begin{array}{l}-1,5 \\
{[-5.6 ; 1.6]}\end{array}$ & 0.02 \\
\hline $\begin{array}{l}\text { Improvement } 10 \% \\
(\mathrm{n}, \%)\end{array}$ & $33(52)$ & $19(32)$ & $16(41)$ & $1(7)$ & $<0.01^{*}$ \\
\hline $\mathrm{VO}_{2} \max$ & $\begin{array}{l}0.2 \\
{[0.0 ; 0.5]}\end{array}$ & $0.1[-0.1 ; 0.3]$ & $0.3[-0.0 ; 0.4]$ & $\begin{array}{l}-0.2 \\
{[-0.6 ; 0.1]}\end{array}$ & $<0.01$ \\
\hline Pain & $\begin{array}{l}-1.1 \\
{[-4.4 ; 0]}\end{array}$ & $-2.2[-4.4 ; 0]$ & $-2.2[-5.5 ; 0]$ & $2.8[-4.4 ; 2.2]$ & 0.67 \\
\hline $\begin{array}{l}\text { Reduction 30\% } \\
(\mathrm{n}, \%)\end{array}$ & $29(46)$ & $20(33)$ & $9(23)$ & $4(29)$ & $0.11^{\ddagger}$ \\
\hline
\end{tabular}

Values are median [IQR] unless stated otherwise

"Changes after 3 months measured as follow-up-baseline

${ }^{\dagger}$ Tested using the Kruskal-Wallis equality-of-populations rank test

*Tested using Pearson's chi-squared
Table 3. Association between clinically relevant improved fitness and clinically relevant reduced pain.

\begin{tabular}{|l|c|c|c|}
\hline Fitness categories & OR & $\mathbf{9 5 \%}$ CI & p \\
\hline Very low (n=63) & 0.74 & $(0.27 ; 2.00)$ & 0.55 \\
\hline Low (n=60) & 0.88 & $(0.29 ; 2.67)$ & 0.82 \\
\hline Medium (n=39) & 1.28 & $(0.35: 4.73)$ & 0.71 \\
\hline
\end{tabular}

The group High/very high could not be analyzed due to inadequate observations ( $\mathrm{n}=13$ )

\section{Discussion}

The present study assessed the association between fitness and pain intensity in patients with LBP. No correlation between baseline fitness and pain scores was detected. The group of patients with the poorest baseline fitness levels also reported the highest baseline pain intensity, but no association between clinically relevant improved fitness and reduced pain intensity could be demonstrated.

The strength of the study was the relatively large study population $(n=176)$. In addition, the risk of assessment bias was considered minimal as changes in fitness was estimated using an objective measurement where judgment from neither the assessor nor the patient was of importance. Pain intensity was assessed using the NRS which is considered a valid and reliable instrument to measure pain intensity in patients with LBP [14].

A limitation of this study was the large group of patients who were lost to follow-up, but as the groups had similar baseline characteristics, the risk of bias due to the dropout is considered minimal, and the dropout has probably not had major influence on the result.

Fitness was estimated using Aastrands cycle test. As the test is submaximal there was a risk of misclassification of the exact fitness. The test is reliable in assessing changes in fitness in LBP patients [18], and the risk of misclassification of changes in fitness was considered small. It is however unknown to which degree a $10 \%$ improvement in fitness is influenced by measurement bias. This could potentially influence the result towards no association. An analysis incorporating an alternative cut off for fitness at $20 \%$ was performed, but this did not alter the results. By incorporating the alternative cut off at $20 \%$ the risk of the improvement being the result of measurement bias and not a clinically relevant improvement was reduced.

The result of this study is consistent with the results presented in three other cross-sectional studies were no correlation between fitness and pain was detected $[7,11,12]$. The studies were of low quality and the results must be interpreted with caution. The association between changes in fitness and changes in pain has been examined in two other studies. The study by Chan et al. found no association [13], but the quality of the study was limited because of deficient blinding and a small study population $(\mathrm{n}=46)$. A study by van der Velde et al. found an association between improved fitness and reduced pain [9], but the results were limited as the study population consisted of patients not responding to conventional therapy and the risk of bias due to a high dropout rate.

A recent published meta-analysis by Meng et al. stated that based on the current literature patients with LBP may benefit from aerobic exercise, as their pain scores had markedly been reduced with a standardized mean difference (SMD) of 0.75 (95 CI: 0.48;1.02). The meta-analysis however also showed that aerobic exercise had no effect on maximum oxygen consumption [19]. Fitness as an approximation of physical capacity may be an insufficient description, since the degree of physical capacity may change, without necessarily reflecting change 
in fitness. In addition, physical capacity also depends on e.g. muscle strength, endurance and motivation, and it should be considered whether physical fitness is the best approximation for physical capacity.

The present study incorporates clinically relevant thresholds based on the conclusion that statistical significance does not necessarily mean clinical importance for the patients [20]. To our knowledge no other studies have examined the association incorporating clinically relevant thresholds. In future studies, it could also be taken into consideration if the clinically relevant changes in pain intensity are pertinent regardless of the patients' pain score at baseline.

The high baseline pain intensity among the group of patients with the poorest fitness levels indicates that further research into the possible association between changes in fitness and changes in pain intensity is needed. However, a possible causal association may be difficult to establish and may only partially be explained by changes in fitness or physical activity, as changes in pain intensity is influenced by multiple factors. In addition, further research is needed to establish an evidencebased threshold of clinically relevant improved fitness.

\section{Conclusion}

In this study, we examined the association between fitness and pain intensity. No correlation between baseline fitness and pain intensity was detected and no association between changes in fitness and pain intensity during a three-month period was found. The clinically relevant thresholds in this study provide an important link between science and clinical practice. Although no association was detected, the use of clinically relevant thresholds in research is likely to be of great interest to researchers as well as clinicians. No other studies have examined the association based on clinically relevant thresholds. The result implies no association between changes in fitness and pain intensity, but based on current evidence, it is not possible to conclude whether an improvement in fitness has an impact on pain intensity.

\section{Ethics approval}

Ethics approval was provided by the Danish Data Protection Agency, number 2006-41-6190, and notified to the Central Denmark Region Committees on Biomedical Research Ethics.

\section{Acknowledgements}

We would like to thank dr. Lone Donbæk Jensen, Aarhus University Hospital, for her attendance in the study.

\section{Conflict of interest}

None stated.

\section{References}

1. Hoy D, March L, Brooks P, Blyth F, Woolf A, et al. (2014) The global burden of low back pain: estimates from the Global Burden of Disease 2010 study. Ann Rheum Dis73:968-974.

2. van Middelkoop M, Rubinstein SM, Kuijpers T, Verhagen AP, Ostelo R, Koes BW, et al. (2011) A systematic review on the effectiveness of physical and rehabilitation interventions for chronic non-specific low back pain. Eur Spine J20:19-39.[crossref]

3. Bakker EW, Verhagen AP, van Trijffel E, Lucas C, Koes BW (2009) Spinal mechanical load as a risk factor for low back pain: a systematic review of prospective cohort studies. Spine (Phila Pa 1976) 34: E281-293. [crossref]

4. Kamper Steven J, Apeldoorn Andreas T, Chiarotto A, Smeets Rob JEM, Ostelo Raymond WJG, et al. (2014) Multidisciplinary biopsychosocial rehabilitation for chronic low back pain. Cochrane Database Syst Rev9: CD000963.[crossref]

5. Hayden JA, van Tulder MW, Malmivaara A, Koes BW (2005) Exercise therapy for treatment of non-specific low back pain. Cochrane Database Syst Rev 20: CD000335. [crossref]

6. Hayden JA, van Tulder MW, Tomlinson G (2005) Systematic review: strategies for using exercise therapy to improve outcomes in chronic low back pain. Ann Intern Med 142: 776-785. [crossref]

7. Smeets RJ, Wittink H, Hidding A, Knottnerus JA (2006) Do patients with chronic low back pain have a lower level of aerobic fitness than healthy controls? are pain, disability, fear of injury, working status, or level of leisure time activity associated with the difference in aerobic fitness level? Spine (Phila Pa 1976)31:90-97.[crossref]

8. Duque I, Parra JH, Duvallet A (2011) Maximal aerobic power in patients with chronic low back pain: a comparison with healthy subjects. Eur Spine J 20: 87-93.[crossref]

9. van der Velde G, Mierau D (2000) The effect of exercise on percentile rank aerobic capacity, pain, and self-rated disability in patients with chronic low-back pain: a retrospective chart review. Arch Phys Med Rehabil 81: 1457-1463.[crossref]

10. Duque I, Parra JH, Duvallet A (2009) Physical deconditioning in chronic low back pain. J Rehabil Med 41: 262-266. [crossref]

11. Rasmussen-Barr E, Lundqvist L, Nilsson-Wikmar L, Ljungquist T (2008) Aerobic fitness in patients at work despite recurrent low back pain: a cross-sectional study with healthy age- and gender-matched controls. J Rehabil Med40: 359-365.[crossref]

12. Wittink H, Michel TH, Sukiennik A, Gascon C, Rogers W (2002) The association of pain with aerobic fitness in patients with chronic low back pain. Arch Phys Med Rehabil 83: 1467-1471.[crossref]

13. Chan CW, Mok NW, Yeung EW (2011) Aerobic exercise training in addition to conventional physiotherapy for chronic low back pain: a randomized controlled trial. Arch Phys Med Rehabil 92:1681-1685.[crossref]

14. Ostelo RW, Deyo RA, Stratford P, Waddell G, Croft P, et al. (2008) Interpreting change scores for pain and functional status in low back pain: towards international consensus regarding minimal important change. Spine 33:90-94. [crossref]

15. Jensen LD, Maribo T, Schiottz-Christensen B, Madsen FH, Gonge B, et al. (2012) Counselling low-back-pain patients in secondary healthcare: a randomised trial addressing experienced workplace barriers and physical activity. Occup Environ Med 69:21-28.[crossref]

16. Textbook of work physiology (1986) Physiological bases of exercise. (3 $3^{\text {rd }}$ ed.), New York: McGraw Hill

17. Astrand I (1960) Aerobic work capacity in men and women with special reference to age. Acta Physiol Scand Suppl 49: 1-92.[crossref]

18. Keller A, Hellesnes J, Brox JI (2001) Reliability of the Isokinetic Trunk Extensor Test, Biering-Sørensen Test, and Åstrand Bicycle Test: assessment of intraclass correlation coefficient and critical difference in patients with chronic low back pain and healthy individuals. Spine26:771-777

19. Meng XG, Yue SW (2015) Efficacy of aerobic exercise for treatment of chronic low back pain: a meta-analysis. Am J Phys Med Rehabil 94: 358-365.[crossref]

20. van Tulder M, Malmivaara A, Hayden J, Koes B (2007) Statistical significance versus clinical importance: trials on exercise therapy for chronic low back pain as example. Spine (Phila Pa 1976) 32:1785-1790.
Copyright: (C2016 Budtz CR. This is an open-access article distributed under the terms of the Creative Commons Attribution License, which permits unrestricted use, distribution, and reproduction in any medium, provided the original author and source are credited. 